\title{
Using Curve-Fitting of Curvilinear Features for Assessing Registration of Clinical Neuropathology with in Vivo MRI
}

\author{
P.P. Laissue, C. Kenwright, S.A. Hojjat, and A.C.F. Colchester \\ Medical Image Computing, University of Kent \\ Canterbury CT2 7PD, United Kingdom
}

\begin{abstract}
Traditional neuropathological examination provides information about neurological disease or injury of a patient at a high-resolution level. Correlating this type of post mortem diagnosis with in vivo image data of the same patient acquired by non-invasive tomographic scans greatly complements the interpretation of any disease or injury. We present the validation of a registration method for correlating macroscopic pathological images with MR images of the same patient. This also allows for 3-D mapping of the distribution of pathological changes throughout the brain. As the validation deals with datasets of widely differing sampling, we propose a method using smooth curvilinear anatomical features in the brain which allows interpolation between widespaced samples. Curvilinear features are common anatomically, and if selected carefully have the potential to allow determination of the accuracy of coregistration across large areas of a volume of interest.
\end{abstract}

\section{Introduction}

For the majority of diseases, histopathology is the 'gold standard' to support and confirm a diagnosis. It is thus important that data derived from non-invasive imaging techniques such as MRI is evaluated with established histopathological data. In the case of neuropathology, correlation of in vivo MR scans of the cerebrum with post mortem histopathological sections of the same brain can be achieved by using a macrotome. This allows cutting thin slices $(30-100 \mu \mathrm{m})$ of the post mortem brain, generating a uniform sampling which exceeds in all dimensions that of an MR scan. However, this process is too costly and time-consuming in the context of clinical neuropathology, where large slices (7-20mm thick) of the fixed brain are cut by hand. The registration and validation method presented here has been specifically developed to enable correlation of a tomographic scan of an intact brain (in vivo or post mortem) with the same brain processed post mortem in a clinical neuropathology laboratory, where the use of a macrotome is not feasible.

In this paper, we first summarise the overall registration approach, which has been presented in parts before $[1,2,3]$. We then focus on its validation.

Validation of registration procedures, a central challenge in biomedical image computing, is required to appraise rigid and especially non-rigid registration. While point-based landmarks (attached markers or natural features) are widely used, they are not applicable where imaging modalities involve thick slices or wide separation 
between slices. In such cases, it is difficult to establish enough point landmarks, which often cannot be localised accurately and, if they lie between slices, may not be imaged at all. Alternatively, volume-based measures (e.g., overlap index, [4]) have been widely used. Information is commonly computed for surfaces from a distance transform, which is integrated over the whole feature. However, these are usually presented as scalar (i.e., non-directional) values, and underestimation in some areas may be compensated by overestimation in others, giving a false sense of accuracy. For these reasons, we developed an approach to validation which uses curvilinear features. Such space curves have already been used to drive registration (e.g., [5]) and have potential advantages. Directional error measures can be calculated orthogonal to the main axis or axes of the feature, providing partial information on the direction as well as the magnitude of the error at any chosen location.

Care is needed over the choice of curves or surfaces. To constrain all the necessary directional parameters, curves or surfaces that cover a good range of orientations and positions must be used. If the natural features curve smoothly then interpolation across substantial slices intervals can be performed, and the smoothed curves can be used for matching or error measurement [6].

Here we present the results of the validation and show that an appropriate choice of smooth curvilinear features in the lateral ventricles (LV) of the brain allows reliable registration estimations with directional information over a substantial part of the field of view.

\section{Methods, Patients and Images}

\subsection{Patients and Images}

Datasets of four patients were studied. In vivo MR scans (IVMR) were made available from earlier clinical investigations and had not been planned as part of this study. In three cases, the quality turned out to be very poor due to clinical acquisition constraints in confused and restless patients. Therefore, post mortem MR scans (PMMR) were acquired and used instead. In the fourth case, both IVMR and PMMR could be used. Slice thickness for all MR datasets was $1.5 \mathrm{~mm}$. Post mortem pathology studies were carried out in a clinical neuropathology laboratory. After fixation in formaldehyde, thick coronal brain slices were cut and from these slices blocks were subsequently removed for microscopic examination. Brain slices were 7-21mm thick. They were put in a specially designed jig with fiducial markers and photographed, in order to obtain the post mortem optical datasets (PMOP).

\subsection{Approach to Registration}

During post mortem processing of the brain, shrinkage, distortion and sometimes movement of loosely connected fragments may occur. Hence, a non-rigid registration approach was essential. Registration was performed using a multi-level processing pipeline with seven steps each driven by different features. For ease of explanation we grouped the steps into three main stages. 


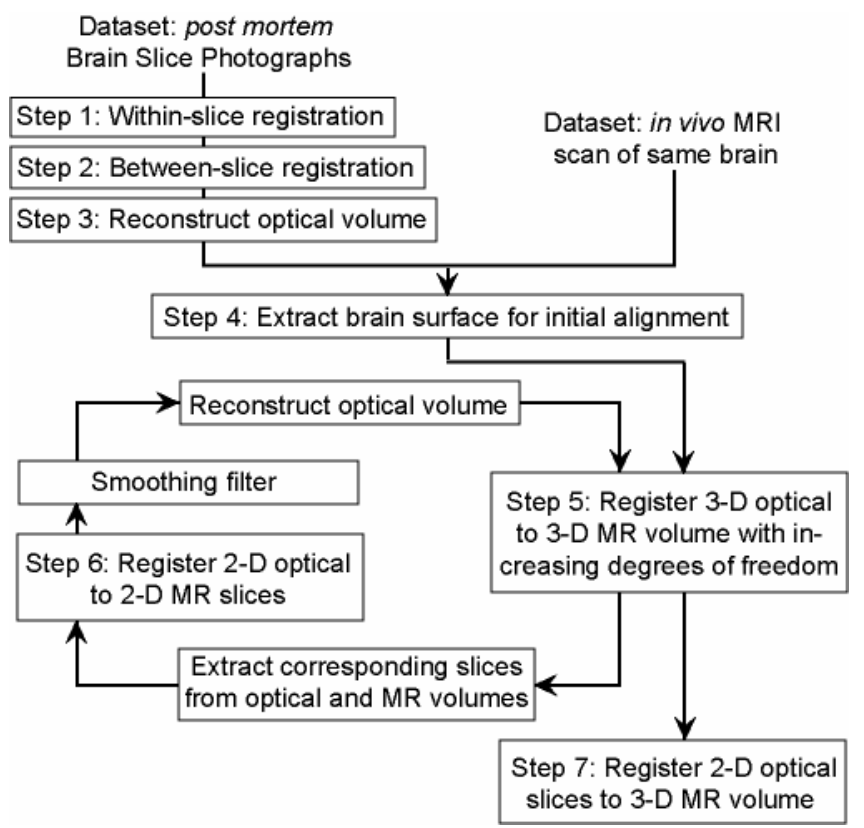

Fig. 1. Overview of the multi-stage algorithm used to register post mortem optical brain slices to the in vivo MR scan of the same brain

\subsubsection{First Stage: Reconstruction of Initial 3-D Optical Volume from the 2-D Brain Slice Photographs}

Step 1 registers the anterior and posterior face of the same slice using fiducial circular markers on the brain jig. The cost function is the 2-D Euclidean distance, optimised by least squares. It is initially rigid, and then relaxed to scaling and then affine transformation. Step 2 is based on grey scale pixel values and registers the anterior and posterior face of adjacent slices. Correlation coefficient and sum of squared residuals are used as cost function, and it is optimised by coarse to fine block matching, using Least Trimmed Squares. The final step in this stage is used to reconstruct the optical volume, selecting a slice near the middle of the slice sequence as the reference. These 2-D ( $\mathrm{x} \& \mathrm{y})$ transformations are propagated forwards and backwards by matrix multiplication. At this stage, slices are assumed equally spaced with the thickness specified by the neuropathologist, and slice faces are assumed parallel, and "z" values are thereby assigned to the face of each slice.

\subsubsection{Second Stage: Registration of MR Data with the Optical Volume}

using iterative refinement through a sequence of global 3-D registrations and local 2$\mathrm{D}$ registrations. For each of the steps, co-registration is initially rigid, then relaxed affine. Step 4 extracts the brain surfaces of optical and MR volume which are coregistered using an Iterative Closest Point algorithm. Step 5 registers the 3-D optical volume with the MR volume based on grey scale voxel values throughout the volumes. A modified correlation ratio [7] is used as cost function, optimised by BrentPowell multi-dimensional gradient descent. Step 6 performs in-plane registration of a 
2-D optical slice with its corresponding 2-D MR slice. Registration objects are again grey scale voxel values, and the correlation coefficient and sum of squared residuals represent the cost function. Block matching and Least Trimmed Squares optimise the registration. The transformation matrices are then smoothed over all slices. After step 6 , the algorithm returns to step 5 for two further iterations, then repeats step 6 .

\subsubsection{Third Stage: Final 2-D Optical Slice to 3-D MR Volume Refinement Allowing Out-of-Plane Movement}

Step 7 is used to correct residual inaccuracies. It is based on grey scale voxel values, with the correlation ratio as cost function, with optical slices being allowed out-ofplane translations, rotations and anisotropic scaling.

\subsection{Validation of Registration}

The registration was initially assessed visually. To quantify the registration, the similarity coefficient of Jaccard (1) was used to determine the amount of overlap between the co-registered IVMR or PMMR (I) and PMOP (J) datasets. It is defined as

$$
P(I, J)=\frac{P(I \cap J)}{P(I \cup J)}
$$

and was determined for the entire brain and for the LV (see Table 1).

In order to provide a directional quantitative estimate of registration error, we used the crest lines of the LV of the brain. A crest line comprises all points on a surface of local maximum curvature and forms a space curve for smooth surfaces in 3-D [8, 9]. In the LV, these crest line features are generally well-defined anatomically and curve smoothly. They also have the advantage that they bend in all three dimensions, so that discrepancies in all main degrees of freedom can be estimated. In each modality, an expert anatomist identified the crest line points in every slice. A smooth 3D space curve was then computed for each modality using non-uniform rational B-splines (NURBs).

In this application, we present the target registration error as distance between the curves. We introduce a mean inter-curve distance $D$ over a set number of intervals $N$, as given by

$$
D=\frac{\sum_{i}^{N}\left|c_{i}-k_{i}\right|}{N}
$$

between the crest lines $c$ and $k$. Intervals were chosen at a subvoxel resolution and from each such interval the shortest 3-D distance to the crest line of the co-registered dataset was computed. These error vectors ensure that the validation is voxel- and slice-independent. Measurements were grouped according to the thickness of the PMOP slices (see Table 2). Registration error between the curves was further assessed by determining the angular component, as described in the Results section. 


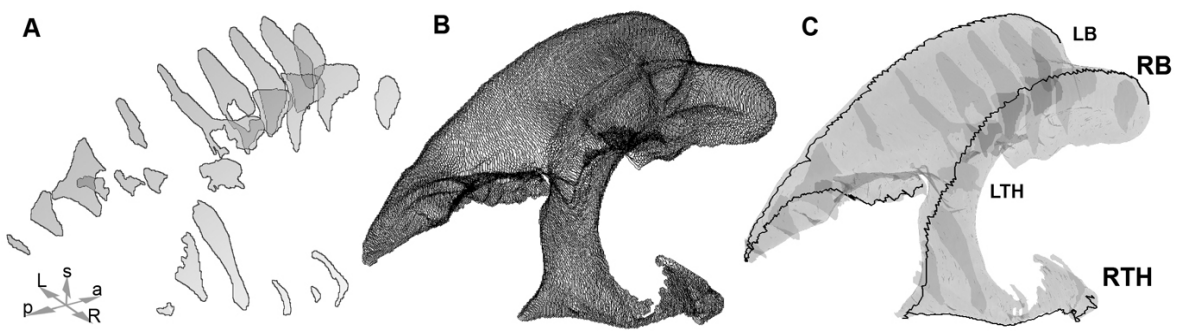

Fig. 2. Isometric projections of the boundaries of the lateral ventricle (LV) as seen in different datasets. A) Outlines of the LV, defined in post mortem optical slices (PMOP). B) Outlines of $\mathrm{LV}$ as reconstructed from in vivo MRI scan (IVMR). C) Combined representation of both modalities. Light grey semitransparent surface: LV reconstructed from MRI; dark grey LV sections from PMOP; black space curves: unsmoothed crest lines of the LV from the IVMR dataset. The cross in the lower left corner indicates p: posterior, a: anterior, L: Left, R: Right, s: superior. Compartments of the LV are indicated by initials: LB: Left Body, RB: Right Body, LTH: Left Temporal Horn, RTH: Right Temporal Horn.

\section{Results}

\subsection{Validation Using Visual Assessment and Jaccard Similarity Coefficient}

Registration was initially validated visually by assessing anatomical correspondence in overlayed images, showing that the outer boundaries correspond well.
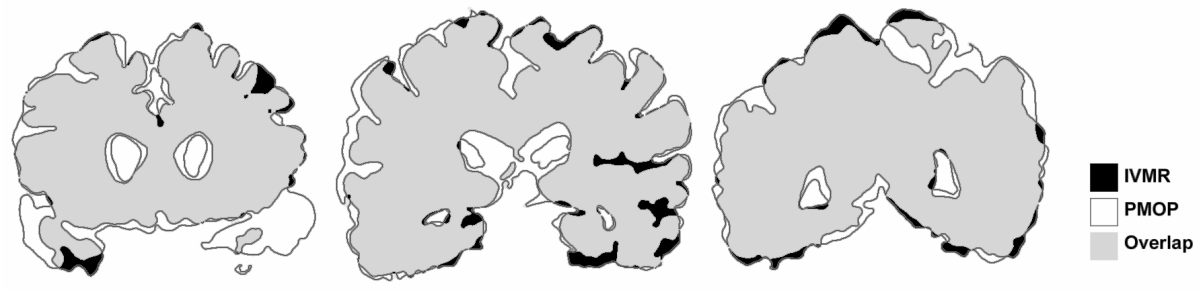

Fig. 3. 2-D visualisation of registration of two 3-D co-registered datasets from Patient unc022. Three representative coronal slices of fourteen are shown, from anterior (left hand slice) to posterior (on the right).

Table 1. Validation of intermodal registration using the Jaccard similarity coefficient ('Jacc.'), as determined for the entire brain ('Br') and the lateral ventricle ('LV'). Left half: Results for the patient with three datasets. Right half shows the results for three other patients for which PMMR and PMOP datasets were used.

\begin{tabular}{|c|cccccc|cc|cc|cc|}
\hline Patient & \multicolumn{4}{|c|}{ unc022 } & \multicolumn{2}{c|}{ unc004 } & \multicolumn{2}{|c|}{ vcjd021 } & \multicolumn{2}{c|}{ vcjd001 } \\
\hline $\begin{array}{c}\text { Data- } \\
\text { sets }\end{array}$ & $\begin{array}{c}\text { IVMR- } \\
\text { PMOP }\end{array}$ & $\begin{array}{c}\text { PMMR- } \\
\text { PMOP }\end{array}$ & \multicolumn{2}{c|}{$\begin{array}{c}\text { IVMR- } \\
\text { PMMR }\end{array}$} & \multicolumn{2}{c|}{$\begin{array}{c}\text { PMMR- } \\
\text { PMOP }\end{array}$} & \multicolumn{2}{c|}{$\begin{array}{c}\text { PMMR- } \\
\text { PMOP }\end{array}$} & \multicolumn{2}{c|}{$\begin{array}{c}\text { PMMR- } \\
\text { PMOP }\end{array}$} \\
\hline Part & $\mathrm{Br}$ & LV & $\mathrm{Br}$ & LV & $\mathrm{Br}$ & LV & $\mathrm{Br}$ & LV & $\mathrm{Br}$ & LV & $\mathrm{Br}$ & LV \\
\hline Jacc. & 0.81 & 0.47 & 0.82 & 0.55 & 0.79 & 0.61 & 0.88 & 0.51 & 0.91 & 0.29 & 0.81 & 0.17 \\
\hline
\end{tabular}




\subsection{Validation of Interpolation of Ventricular Crest Lines between Widely Spaced Slices}

MRI data provided a well sampled model, allowing us to validate the process of interpolation which was applied in the main study to the optical data with widely spaced slices. From the MRI data only, the points in each slice that lay on the ventricular crest lines were identified at intervals chosen to match the spacing of the optical data. This meant using every $27^{\text {th }}$ slice, each separated by $10 \mathrm{~mm}$. These points were used to create smooth interpolated space curves. The interpolated space curves were then compared with the actual locations identified in every adjacent MRI slice. The average within-slice deviation of the true and predicted crest points was 1.8 pixels, corresponding to $1.8 \mathrm{~mm}$, and the directional error varied around zero, i.e., there was no substantial bias.

\subsection{Validation of Multi-modality Registration}

Within subjects, ventricular crest lines, extracted separately from MRI and from optical data, were compared to evaluate the overall registration process. The magnitude of the error vectors (see section 2.3) had a mean value of $5.4 \mathrm{~mm}(\mathrm{RTH}=4.1, \mathrm{LTH}=3.6$, $\mathrm{RB}=6.8, \mathrm{LB}=7.1$ ) but there was a wide range, from $1.56 \mathrm{~mm}$ to $12.42 \mathrm{~mm}$. The errors were generally greater in the posterior half (i.e. the occipital horn) of the LV (see Table 2). The direction of the error, from the IVMR to the PMOP crest lines, varied systematically. For the ventricular bodies, the predominant direction of the error was inferior (Fig. 4), while for the temporal horns the predominant direction was medial and superior. Thus, for these ventricular features, the optical data appears shrunken relative to the MRI data.

Table 2. Target registration errors (TRE) and angular deviation of the LV crest lines of patient unc022, averaged within segments as defined in the diagram (left). Segments match the PMOP slice spacing. Measurement is from the IVMR to the PMOP dataset. TRE is in mm, angles $\alpha$ and $\beta$ are indicated in degrees (rounded). For explanation and illustration of angles, see Figure 4 on the next page.

\begin{tabular}{|c|c|c|c|c|c|c|c|c|c|c|c|c|c|}
\hline \multirow{4}{*}{$L T H$} & \multirow{2}{*}{$\begin{array}{l}\angle B \\
A\end{array}$} & $R E$ & & & & & & & & & & & \\
\hline & & A & & & & & & & & & & & \\
\hline & B & B & RTH & & & & & & $\mathrm{mpol}$ & $\mathrm{l} \mathrm{Ho}$ & & & \\
\hline & D & D & $D$ & & & & $\mathrm{t}(\mathrm{LT}$ & & & & ight & RTH & \\
\hline 5 & $E$ & $E$ & E & Segment & $\mathrm{D}$ & $\mathrm{E}$ & $\mathrm{F}$ & $\mathrm{G}$ & $\mathrm{H}$ & $\mathrm{D}$ & $\mathrm{E}$ & $\mathrm{F}$ & $\mathrm{G}$ \\
\hline $\mathbf{F}$ & F & $\mathbf{F}$ & $F$ & TRE & 7.1 & 6.2 & 2.1 & 1.6 & 0.9 & 3.1 & 3.7 & 4.4 & 5.2 \\
\hline G & G & G & G & $\alpha$ & 15 & -13 & 10 & 18 & -8 & 7 & 1 & 18 & 27 \\
\hline H & H & & & $\beta$ & 32 & 36 & 21 & 17 & -31 & 29 & -2 & 7 & 22 \\
\hline
\end{tabular}

\begin{tabular}{|c|cccccccc|ccccccc|}
\hline \multicolumn{11}{|c}{} & \multicolumn{11}{c|}{ Left (LB) } & \multicolumn{1}{c|}{ Body } \\
Segment & A & B & C & D & E & F & G & H & A & B & C & D & E & F & G \\
\hline TRE & 3.4 & 4.4 & 6.8 & 7.5 & 8.9 & 10.2 & 8.9 & 6.4 & 2.5 & 3.3 & 5.2 & 7.7 & 11.7 & 12.4 & 4.9 \\
$\alpha$ & -22 & -7 & 1 & 7 & 30 & 36 & 44 & 42 & -26 & -11 & -3 & 11 & 31 & 30 & 7 \\
$\beta$ & -33 & -26 & -46 & -73 & -51 & -48 & -46 & -42 & -58 & -73 & -84 & -78 & -56 & -51 & -54 \\
\hline
\end{tabular}




\section{Discussion}

The registration algorithm is designed to deal with variations in slice thickness and other errors that can occur as a result of the specimen preparation and earlier processing. Validation of the registration results for patients with good to moderate quality MRI shows an acceptable degree of correlation, confirming that it may be used routinely in neuropathology laboratories where thick slices are cut.

The MRI scans do require a moderate degree of quality to fulfill the needs of this registration procedure. In this study, IVMR scans with $6.5 \mathrm{~mm}$ slice thickness provided insufficient information to find correct global minima when registering the outlines of the brain. By contrast, all PMMR scans with $1.5 \mathrm{~mm}$ and one IVMR with $1 \mathrm{~mm}$ slice thickness could be used for registration with their corresponding PMOP datasets.

The validation of this study can be compared to only few others. Singh and coworkers [10] evaluated their method of registering MRI datasets with $5 \mathrm{~mm}$ thick PMOP slices of the same brain and determined an error ranging from 3.4 to $10.9 \mathrm{~mm}$ with an average $7.5( \pm 2.7) \mathrm{mm}$. Our results of $5.4( \pm 0.7) \mathrm{mm}$ compare favourably. Crum et al. [4] evaluated different non-rigid registration methods for interindividual registration of MRI datasets by analysing the entire brain and various substructures. The most successful method, based on B-Splines, performed exceptionally well for registering the $\mathrm{LV}$, with a Jaccard similarity coefficient of 0.92 for the whole brain and 0.78 for the LV. Averaged over all specimens used here, our method achieves 0.84 for the whole brain and 0.43 for the LV.

Although the ventricles are extended in space, they still lie relatively deeply in the cerebrum, while our automated registration procedures mainly used more superficial data. Consistent with this, visual inspection in general suggested that registration was most accurate with the superficial structures, and became less reliable with the deep structures. Therefore, our error measures are likely to reflect the least favourable aspect of the overall accuracy. They also suggest that the shrinkage of the LV has not been fully compensated by the automated non-rigid registration procedure used.

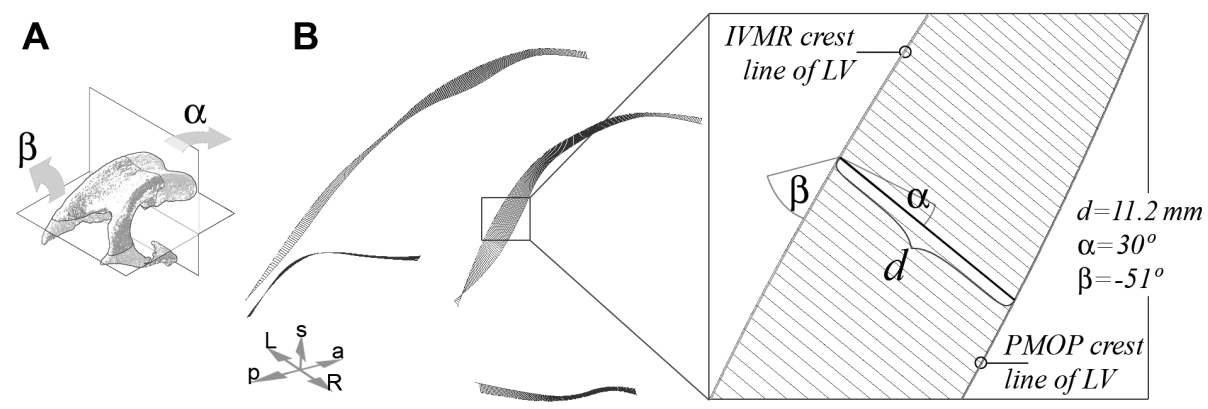

Fig. 4. Crest lines of the LV with shortest-distance error lines constructed between them. A) Isometric view of the LV showing the choice of angles. $\alpha$ designates anterior rotation relative to the coronal plane (negative values $=$ posterior rotation) and $\beta$ elevation relative to the axial plane (negative values $=$ depression). $\mathrm{B}$ ) Isometric view of the crest lines and the distance and angle measurements between them. The box on the RB is magnified at right. Error lines are grey, with measurements for one example black, indicated on the right side of the box. 
We show that the crest lines of the LV are good features to validate the registration of datasets with widely differing resolutions. They allow us to assess registration errors that are difficult to determine with other validation methods used in such cases. The method presented here allows us to examine the quality of registration in three dimensions with quantification of directional as well as scalar errors.

Curvilinear features are quite common in many anatomical contexts and have the potential to be used more widely. To justify interpolating between widely spaced slices, two key aspects should be respected. Firstly, features chosen for interpolation should naturally have smooth curves. Secondly, the interpolation method used needs to be validated in the specific context, as we have done here.

While we have only used crest lines of the LV, we are currently evaluating other cerebral structures. There is a whole inventory of features within the brain that conforms to the requirements. This is not only valid for the brain. In many other organs, crest line or tubular structures such as blood vessels can be found to which this method may be applied.

\section{References}

1. Colchester, A.C.F., Ourselin, S., Zhu, Y., Bardinet, E., He, Y., Roche, A., Al-Sarraj, S., Nailon, B., Ironside, J., Ayache, N.: 3-D Reconstruction of Macroscopic Optical Brain Slice Images. Med. Image Comp. \& Comp.-Assist Intervent 1935, 95-105 (2000)

2. Bardinet, E., Colchester, A.C.F., Roche, A., Zhu, Y., He, Y., Ourselin, S., Nailon, B., Hojjat, S.A., Ironside, J., Al-Sarraj, S., Ayache, N., Wardlaw, J.: Registration of Reconstructed Post Mortem Optical Data with MR Scans of the Same Patient. Med. Image Comp. \& Comp-Assist Intervent 2208, 957-965 (2001)

3. Kenwright, C., Bardinet, E., Hojjat, S.A., Malandain, G., Ayache, N., Colchester, A.C.F.: 2-D to 3-D Refinement of Post Mortem Optical and MRI Co-registration. Med. Image Comp. \& Comp-Assist Intervent 2879, 935-944 (2003)

4. Crum, W.R., Rueckert, D., Jenkinson, M., Kennedy, D.N., Smith, S.M.: A framework for detailed objective comparison of non-rigid registration algorithms in neuroimaging. Med. Image Comp. \& Comp-Assist Intervent 3216, 679-686 (2004)

5. Subsol, G., Thirion, J.P., Ayache, N.: A scheme for automatically building threedimensional morphometric anatomical atlases: application to a skull atlas. Med. Image Analysis 2, 37-60 (1998)

6. Gueziec, A., Ayache, N.: Smoothing and matching of 3-D space curves. Int. J. Comp. Vis. 12(1), 79-104 (1994)

7. Roche, A., Malandain, G., Pennec, X., Ayache, N.: The correlation ratio as a new similarity measure for multimodal image registration. Med. Image Comp. \& Comp.-Assist Intervent 1496, 1115-1124 (1998)

8. Monga, O., Ayache, N., Sander, P.T.: From voxel to intrinsic surface features. Image Vis Comp. 10, 403-417 (1992)

9. Thirion, J.P., Gourdon, A.: The 3D marching lines algorithm. Graph Models Image Process. 58, 503-509 (1996)

10. Singh, M., Rajagopalan, A., Zarow, C., Zhang, X.-L., Kim, T.-S., Hwang, D., Lee, A.-Y., Chui, H.: From Human MRI to Microscopy: Co-registration of Human Brain Images to Postmortem Histological Sections. Nucl. Sci. Symp. Conf. Rec. 4, 1982-1985 (2006) 